\title{
Les sols du bassin d'Aubure (Haut-Rhin): caractérisation et facteurs de répartition
}

\author{
Y. LEFEVRE \\ INRA, Laboratoire "Sols et Nutrition", Centre de Recherches de Nancy, \\ Champenoux, 54280 Seichamps
}

\begin{abstract}
Summary
The soils in the Aubure catchment: identification and parameters involved in their distribution
\end{abstract}

The Aubure catchment (Vosges, East of France) has been choosen to study the geochemistry of a forested ecosystem.

The first step was to establish a large scale cartography of the soils in the catchment.

Morphological, physico-chemical and chemical analysis were done : parameters involved in the soil distribution were identified.

Results show that the chemical composition of the bedrock greatly influences the pedological processes : for example in this climatical situation, gneiss inhibits podzolisation. Topography and exposure play a secondary but constant role in soil distribution in the landscape.

Key words : Catchment, soil distribution, acidic soils.

\section{Résumé}

Le bassin versant d'Aubure (Haut-Rhin) a été retenu pour étudier la dynamique des éléments minéraux dans un écosystème forestier.

La première étape de cette étude fut l'établissement d'une cartographie précise de la couverture pédologique à grande échelle.

La caractérisation morphologique et physico-chimique des profils a été réalisée ; les grands facteurs responsables de leur répartition dans le paysage sont envisagés.

Les résultats montrent que la composition chimique du substrat influence fortement l'évolution pédologique : à cet étage climatique, la présence de gneiss inhibe la podzolisation.

Au rôle primordial de la roche mère s'ajoute ceux de la topographie et de l'exposition comme facteurs de répartition des sols dans le paysage.

Mots clés : Bassin versant, couverture pédologique, sols acides. 


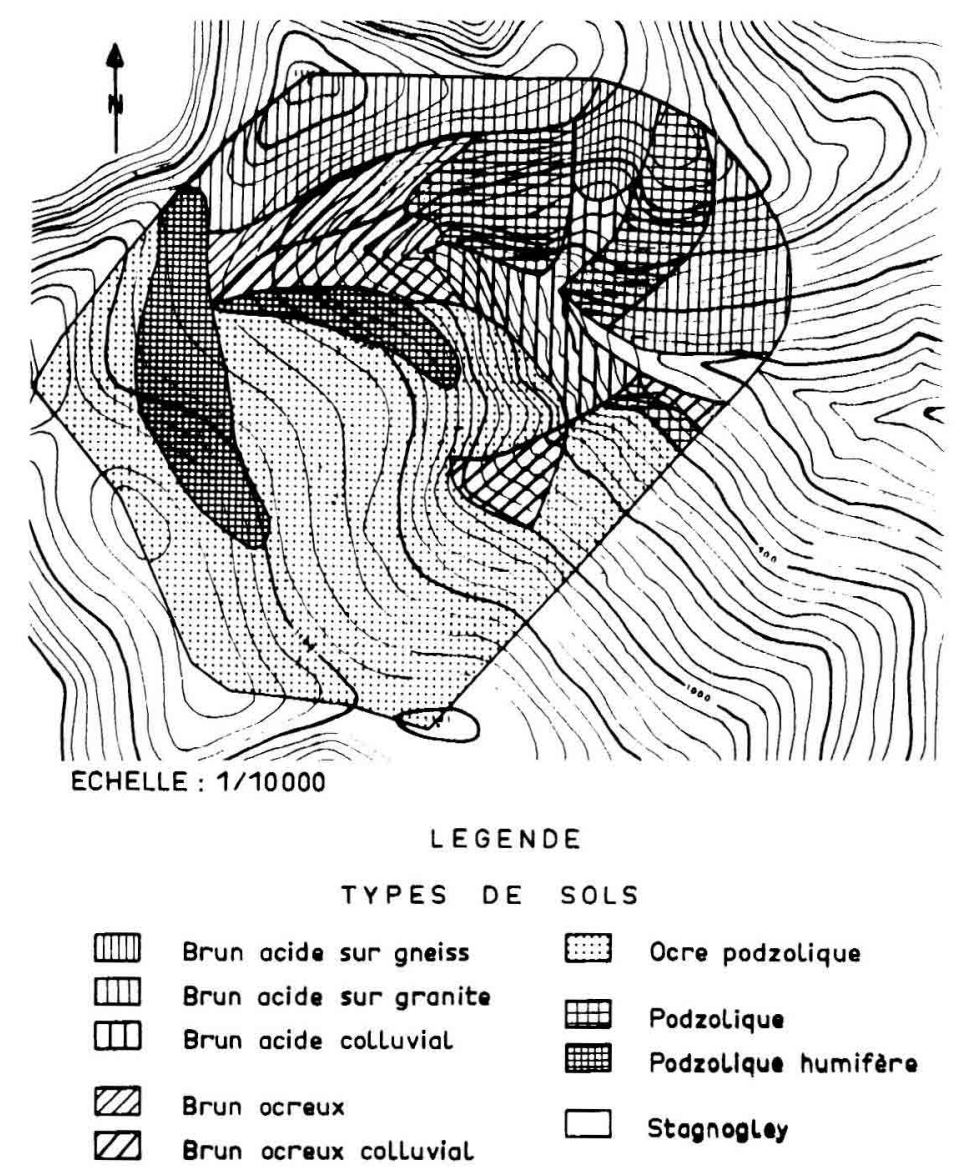

ANNeXe

Bassin versant d'Aubure

Carte pédologique

Annex : Pedological map of the Aubure Catchment. 


\section{Introduction}

Un bassin versant des Vosges alsaciennes a été retenu pour l'étude de la dynamique des éléments minéraux dans un écosystème forestier en milieu acide. Une bonne connaissance des différents compartiments et en particulier des sols est nécessaire. Nous en avons réalisé une cartographie précise à grande échelle, ainsi qu'une caractérisation analytique.

Les principes de l'évolution des sols à l'étage montagnard vosgien sont définis par Souchier (1971). Pour cet auteur, parmi les facteurs écologiques responsables de la pédogénèse, les facteurs stationnels et en particulier la roche mère (par sa teneur en fer et en argile) et secondairement la topographie, jouent un rôle déterminant. Dans ce travail, nous nous sommes intéressés à l'application de ces principes à une échelle plus fine, et nous avons recherché les facteurs explicatifs de l'organisation pédologique dans le bassin en cause.

\section{Matériel et méthode}

\subsection{Cadre de l'étude}

Le bassin versant du Strenbach est situé sur la commune d'Aubure (Haut-Rhin), à l'extrémité Est du massif du Brézouard. Son relief est caractéristique des Hautes-Vosges alsaciennes. Entre des sommets anciens arrondis, le ruisseau Strenbach occupe une profonde entaille d'origine tectonique remaniée par les phénomènes périglaciaires, ce qui explique la forte dénivellation entre les sommets $(1100 \mathrm{~m})$ et le seuil $(880 \mathrm{~m})$. Le climat est de type océanique à tendance montagnarde $(1200 \mathrm{~mm}$ de précipitations dont 15 p. 100 sous forme de neige).

La zone étudiée, d'une superficie d'environ 155 hectares, est essentiellement occupée par la forêt (forêt communale d'Aubure). Les peuplements sont composés à plus de 60 p. 100 par de l'épicéa, essence qui a été fortement étendue depuis la fin du siècle dernier (O.N.F., 1981). Le hêtre et le sapin sont également représentés dans la hêtraie d'altitude et les hêtraies sapinières.

Le bassin versant repose dans sa majorité sur le granite du Brézouard. Ce granite intrusif, d'origine magmatique, est un des plus pauvres du massif vosgien, comme le montre l'analyse de SAAvERDRA et al. (1973) (en p. cent) : $\mathrm{SiO}_{2}: 73,2 ; \mathrm{Al}_{2} \mathrm{O}_{3}: 14,7$; $\mathrm{Fe}_{2} \mathrm{O}_{3}: 1,2 ; \mathrm{MgO}: 2,7 ; \mathrm{CaO}: 0,5 ; \mathrm{Na}_{2} \mathrm{O}: 2,95 ; \mathrm{K}_{2} \mathrm{O}: 5,0$. Deux faciès se différencient: le faciès des leucogranites à deux micas dans le versant nord, celui des leucogranites à cordiérite et muscovite dans le versant sud (DAHIRE, 1988). Les teneurs en fer de ces deux faciès sont très voisines mais la présence de filonnets hématitiques dans le leucogranite du versant sud donne une teinte rougeâtre aux matériaux d'altération. En crête de ce même versant sud affleure un gneiss à sillimatite de la série de Sainte-Marie-aux-Mines. La présence de cette roche, relativement plus riche en fer et magnésium (données de Eller, 1961 (en p. cent) : $\mathrm{SiO}_{2}: 56,8 ; \mathrm{Al}_{2} \mathrm{O}_{3}: 21,6 ; \mathrm{Fe}_{2} \mathrm{O}_{3}$ : 7,$\left.8 ; \mathrm{MgO}: 3,8 ; \mathrm{CaO}: 0,5 ; \mathrm{Na}_{2} \mathrm{O}: 1,0 ; \mathrm{K}_{2} \mathrm{O}: 4,4\right)$ modifie la composition des arènes dans la zone de contact. 


\subsection{Cartographie - Analyse des sols}

Nous avons choisi une méthode d'observation sur un réseau à maille carrée de $100 \mathrm{~m}$ de côté. A chaque point sont observés les principaux caractères morphologiques des horizons, en retenant les critères les plus spécifiques d'évolution sur roche mère acide.

Dans chaque grande unité un profil fait l'objet de déterminations analytiques selon les protocoles habituels décrits par Duchaufour (1979).

\section{Résultats}

\subsection{Caractérisation des sols}

Les sols cartographiés appartiennent principalement à deux classes : classe des sols bruns et classe des sols podzolisés.

Tous les sols présentent un caractère acide marqué ( $\mathrm{pH}$ inférieur à 4) et une grande pauvreté chimique (taux de saturation inférieur à $10 \mathrm{p} .100$ en alcalins et alcalino-terreux, forte proportion de cations acides en particulier de l'aluminium).

Leur degré d'évolution pédogénétique offre une gamme étendue ; ceci est bien traduit par les indices de redistribution du fer et de l'aluminium libres, comparés aux normes définies par Souchier (1971) (tabl. 1).

Les textures sont à nette dominance sableuse, très grossière dans le versant nord (10 p. 100 d'argile), plus équilibrée dans le versant sud (17 p. 100 d'argile).

\section{TABLEAU 1}

Coefficient $K$ de redistribution du fer (méthode $D E B$ ) et de l'aluminium (méthode Tamm) : valeurs théoriques et valeurs observées.

Redistribution (coefficient $K$ ) for iron (evaluated from $D E B$ method) and for aluminium (evaluated from Tamm method) : theorical values from Souchier (1971) and observed values.

\begin{tabular}{|c|c|c|c|c|c|}
\hline & Coefficient de redistribution & $\begin{array}{l}\text { Sols bruns } \\
\text { acides }\end{array}$ & $\begin{array}{l}\text { Sols bruns } \\
\text { ocreux }\end{array}$ & $\begin{array}{l}\text { Sols ocres } \\
\text { podzoliques }\end{array}$ & $\begin{array}{l}\text { Sols } \\
\text { podzoliques } \\
\text { et podzols }\end{array}$ \\
\hline $\mathrm{K} \mathrm{Al}$ & $\begin{array}{l}\text { Souchier } 1971 \ldots \ldots \ldots \\
\text { notre étude } \ldots \ldots \ldots \ldots \ldots \ldots\end{array}$ & $\begin{array}{l}=1 \\
0,8\end{array}$ & $\begin{array}{c}1,1 \text { à } 2 \\
1,8\end{array}$ & $\begin{array}{l}2 \text { à } 3,5 \\
2,5 \text { et } 4,2\end{array}$ & $\begin{array}{l}2,5 \text { à } 9 \\
5,3 \text { et } 3,4\end{array}$ \\
\hline $\mathrm{K} \mathrm{Fe}$ & $\begin{array}{l}\text { Souchier } 1971 \ldots \ldots \ldots \\
\text { notre étude } \ldots \ldots \ldots \ldots \ldots\end{array}$ & $\begin{array}{l}=1 \\
0,8\end{array}$ & $\begin{array}{c}1,1 \text { à } 1,4 \\
1,4\end{array}$ & $\begin{array}{l}1,4 \text { à } 1,8 \\
2,4 \text { et } 2,6\end{array}$ & $\begin{array}{l}1,8 \text { à } 5 \\
3,2 \text { et } 2,6\end{array}$ \\
\hline
\end{tabular}

$\mathrm{Kx}=\frac{2 \mathrm{x} \text { teneur en l'élément } \mathrm{x} \text { dans l'horizon } \mathrm{B}}{\mathrm{S} \text { teneurs en l'élément } \mathrm{x} \text { dans les horizons } \mathrm{A}_{1} \text { et } \mathrm{C}}$ 


\subsection{Répartition des sols}

Nous observons sur la carte représentée en annexe une dissymétrie importante entre les deux versants. L'homogénéité relative des sols du versant nord, à podzolisation moyenne, s'oppose à une distribution plus complexe et une extension un peu surprenante des sols bruns sur le versant sud.

\section{Discussion : Facteurs de répartition des sols dans le paysage}

La présence de l'affleurement de gneiss en crête du versant sud explique l'extension des sols bruns dans cette zone. Le gneiss, par sa richesse en minéraux altérables, comme en témoigne la forte teneur en fer libre de ces profils, ainsi que sa texture plus fine, inhibe les processus de podzolisation (Souchier, 1971). De la même façon, la moindre évolution des sols de la partie médiane du versant sud par rapport à l'autre versant est bien en accord avec la plus forte teneur en argile de sa roche mère.

A ce rôle primordial de la roche mère s'ajoute celui de la topographie, par sa contribution à l'état du matériau et au régime hydrique. Plusieurs auteurs, Legros \& CABidoche (1979), Dambrine (1985), soulignent l'importance de ces facteurs sur la répartition des sols dans les Pyrénées et les Alpes. Ils proposent une typologie générale, qui n'est que partiellement adoptable ici compte tenu de la différence d'échelle. Toutefois nous nous baserons sur les éléments de cette typologie, qui semblent applicables en fonction de nos propres observations, pour proposer le schéma suivant :

\begin{tabular}{|c|c|c|c|c|c|c|}
\hline \multirow{3}{*}{$\begin{array}{c}\text { Versant sud } \\
\Longrightarrow \text { fourniture } \\
\text { hydrique normale }\end{array}$} & $\begin{array}{l}\text { - partie supérieure } \\
\text { (proximité gneiss) }\end{array}$ & + & $\begin{array}{l}\text { pente forte (stabilité } \\
\text { faible) }\end{array}$ & $\Longrightarrow$ & $\begin{array}{l}\text { rajeunissement et } \\
\text { enrichissement du } \\
\text { matériau }\end{array}$ & sol brun acide \\
\hline & - partie médiane & + & pente moyenne & $\Longrightarrow$ & $\begin{array}{l}\text { meilleure stabilité } \\
\text { du matériau }\end{array}$ & $\Longrightarrow$ sol brun ocreux \\
\hline & - partie inférieure & $\begin{array}{l}t \\
t\end{array}$ & $\begin{array}{l}\text { pente forte } \\
\text { matériau très } \\
\text { fragmenté (arène } \\
\text { profonde) }\end{array}$ & $\Longrightarrow$ & $\begin{array}{l}\text { drainage oblique } \\
\text { important }\end{array}$ & $\Longrightarrow$ sol podzolique \\
\hline & $\begin{array}{l}\text { - majorité du } \\
\text { versant }\end{array}$ & + & pente faible & + & $\begin{array}{l}\text { matériau peu } \\
\text { fragmenté }\end{array}$ & $\Longrightarrow$ sol ocre podzolique \\
\hline $\begin{array}{c}\text { Versant nord } \\
\text { (+ froid + humide })\end{array}$ & & & & & & \\
\hline $\begin{array}{c}\Longrightarrow \text { foumiture } \\
\text { hydrique accentuée }\end{array}$ & $\begin{array}{l}\text { - zone particulière } \\
\text { à mi-versant }\end{array}$ & + & $\begin{array}{l}\text { pente légèrement } \\
\text { plus forte }\end{array}$ & + & $\begin{array}{l}\text { apport hydrique } \\
\text { important } \\
\text { proximité du niveau } \\
\text { des sources }\end{array}$ & $\Longrightarrow \underset{\text { Sol podzolique }}{\text { humifère }}$ \\
\hline
\end{tabular}

On remarque qu'une pente forte, qui se traduit généralement par un sol moins évolué, peut au contraire favoriser l'évolution du sol lorsqu'il y a présence d'un matériau très fragmenté, car elle accentue le drainage oblique. 


\section{Conclusion}

Le bassin versant du Strenbach présente une gamme d'évolution et une organisation pédogénétique plus complexe que ne peut le relater la carte pédologique à petite échelle (feuille de Saint-Dié). Nos résultats montrent qu'au rôle primordial de la roche mère (inhibition de la podzolisation liée au gneiss riche en fer) s'ajoute ceux de la topographie et de l'exposition (degré de pente et fourniture hydrique) comme facteurs explicatifs de la répartition des sols.

L'hétérogénéité du bassin n'implique pas obligatoirement des différences fondamentales dans le fonctionnement biogéochimique des deux versants. Il reste néanmoins important que la cartographie réalisée soit bien prise en compte lors de l'établissement du bilan global.

Reçu le 29 septembre 1987.

Accepté le 3 juin 1988.

\section{Références bibliographiques}

DAHiRe M., 1988. Granites et leucogranites paralumineux du Brézouard et du Bilstein (Vosges moyennes): caractères pétrographiques, géochimiques et minéralogiques. Thèse Université de Nancy I, 158 p. + annexes.

Dambrine E., 1985. Contribution à l'étude de la répartition et du fonctionnement des sols de haute montagne, massif des Aiguilles Rouges et du Mont Blanc. Thèse Université de Paris VII, 284 p. + annexes.

Duchaufour Ph., Souchier B., 1979. Pédologie. Tome 2. Constituants et propriétés du sol. Masson Ed., 459 p.

Eller J.P., 1961. Les gneiss de Sainte-Marie-aux-Mines et les séries voisines. Mém. Serv. Carte Géol. Alsace Lorraine, $19,160 \mathrm{p}$.

FaIvre P. \& Gury M., 1978. Notice de la carte pédologique de la France au 1/100000. Les HautesVosges, p. 99 à 135 .

Legros J.P., CABidoche Y.M., 1977. Les types de sols et leur répartition dans les Alpes et les Pyrénées cristallines. Doc. de Cartographie Ecologique, vol. XIX, 1-19.

O.N.F., Centre de Ribeauvillé (Subdivision de Sainte-Marie-aux-Mines, 1981). Procès-verbal de révision d'aménagement de la forêt communale d'Aubure.

SaAvedra J., Roche H. de la, Leterrier J., \& Pei.lmero E., 1973. Essai de typologie géochimique de quelques granites à deux micas des Vosges moyennes. Bull. Soc. Géol. Fr. (7), XV, n” 5-6, p. 541554.

Sovchier B., 1971. Evolution des sols sur roches cristallines à l'étage montagnard (Vosges). Thèse de Doctorat d'Etat, Univ. de Nancy I, $130 \mathrm{p}$. 